\title{
Luz , desmaterialización y transversalidad narrativa: la instalación artística de luz contemporánea y la catedral gótica
}

Light, dematerialization and narrative transversality: the artistic installation of contemporary light and the gothic catedral

Leticia Crespillo Marí

Departamento de Historia del Arte, Universidad de Málaga, España (makiabela@gmail.com)

Recibido el 20 de mayo de 20xx; revisado el 27 de mayo de 2017; aceptado el 30 de mayo de 2017; publicado el 01 de julio de 2017

RESUMEN: La luz como recurso estético es capaz de construir ambientes abstractos y utópicos que representan metáforas visuales que han de ser experimentadas a través de la vivencia corporal y la participación activa del espectador. A través del sistema de iluminación, la obra se convierte en un ritual y se reafirma como un acontecimiento y extensión de un concepto mental que rompe los límites entre lo real y lo imaginario, materializándose artísticamente a través de luz, color, volumen y movimiento. Esto ocurre en dos casos concretos como son la instalación de luz contemporánea y la catedral gótica que, a pesar de su distancia histórica en el tiempo, utilizan este recurso de una manera muy similar.

PALABRAS CLAVES: luz, color, instalación artística, arte contemporáneo, catedral gótica, estética, metáfora visual.

ABSTRACT: This study settles an aesthetic hypothesis regarding the use of light as a language itself, able to create dematerialized and procedure-based spaces in two specific cases, the artistic installation of contemporary light and the Gothic Cathedral. In spite of its historical distance in time, both spaces use a very similar luminescent system, able to build an abstract and utopic ambience, which represents visual metaphors, lived throughout the corporal experience and the active participation of the spectators. Here, the work piece becomes a ritual that reestablishes itself as an event and as an extension of a mental concept breaking the boundaries between reality and what it is imagined. Light, color, volume and movement materialize it all.

KEY WORDS: Light, color, artistic installation, contemporary art, Gothic Cathedral, aesthetics, visual metaphor. 
El término «desmaterialización» quizás sea el que mejor ayude a entender ese desplazamiento que se produce desde el objeto artístico hacia una idea, comportamiento o actitud, ampliando aún más el carácter heterogéneo del que, ya de por sí, goza la práctica artística. La obra se convierte en un concepto que se expande más allá de su propia fisicidad, transformando la manera en la que se percibe, contempla, recibe y experimenta. Los sistemas «no visuales» restan énfasis al aspecto físico de la obra de arte, rompen con la forma de percepción ojocentrista tradicional y la redefinen en un sentido más amplio como transportadora de significados ligados a la propia vida.

En este ámbito, la luz convierte estos sitios en lugares de experimentación donde la mente y el cuerpo, a través de la compresión y el movimiento, descubren un espacio total significante que rodea al espectador y que desemboca en una especie de «umbral conceptual», donde lo físico deja de ser importante y la desmaterialización se rinde a la acción. Un lugar que se vive, se manipula, se expande y se reflexiona a través de la participación, la actividad cognoscitiva pura y la actitud activa del espectador que busca profundizar en esa metáfora estructurada en un plano sensorial diferente a través de luz, el color y los volúmenes [1].

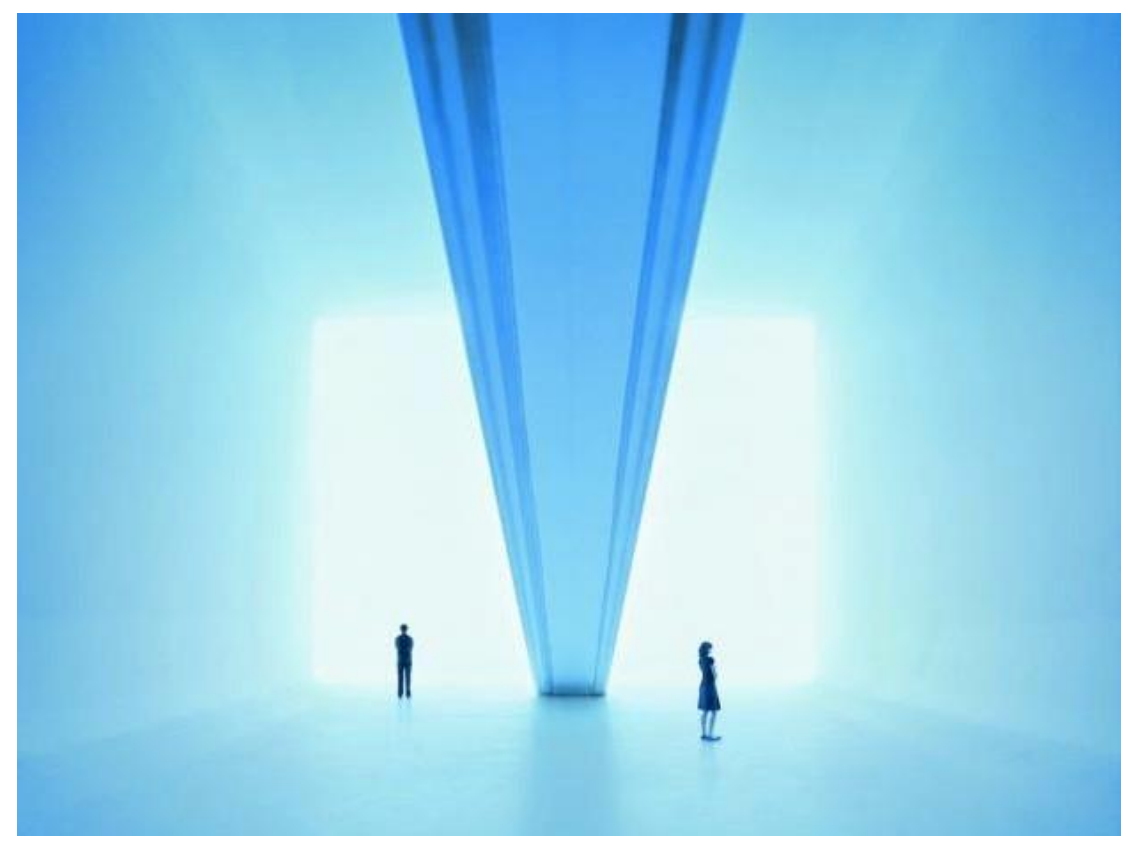

[Fig.1] James Turrell- Bridget's Bardo (2008). Fuente: wikiart.org 


\section{La obra como metáfora y la luz como lenguaje: el proceso mental hacia la construcción de la idea significante}

\section{El espacio de luz contemporáneo ${ }^{1}$}

El espacio contemporáneo es aquel donde el espectador se introduce y lo concibe como parte de su vivencia, como una experiencia frente a la concepción ojocentrista que había predominado con anterioridad durante mucho tiempo. Este espacio se practica, comparte y experimenta a través de la acción activa del espectador que se proyecta en él (Méndez, 2013: 131-132).

Esta situación se evidencia en diversas construcciones que comenzaron por los años 70 y se desarrollaron posteriormente en torno al uso de la luz como en algunas instalaciones de Dan Flavin, James Turrell, Olafur Eliasson o Bruce Nauman. El sujeto transita en la obra y se desenvuelve en esos espacios que investiga y explora, donde el interés reside en esa ampliación del concepto que requiere una mayor atención del sujeto que completa la obra. El movimiento permite esa búsqueda conceptual que reconfigura la relación con el espacio que dialoga con el espectador, permitiéndole acceder a ese espacio de introspección [2]. El espacio se vuelve discurso y narración de un acontecimiento que trasciende lo estrictamente artístico: se niega la materia y la sensación se convierte en idea, energía y movimiento (Méndez, 2013: 133).

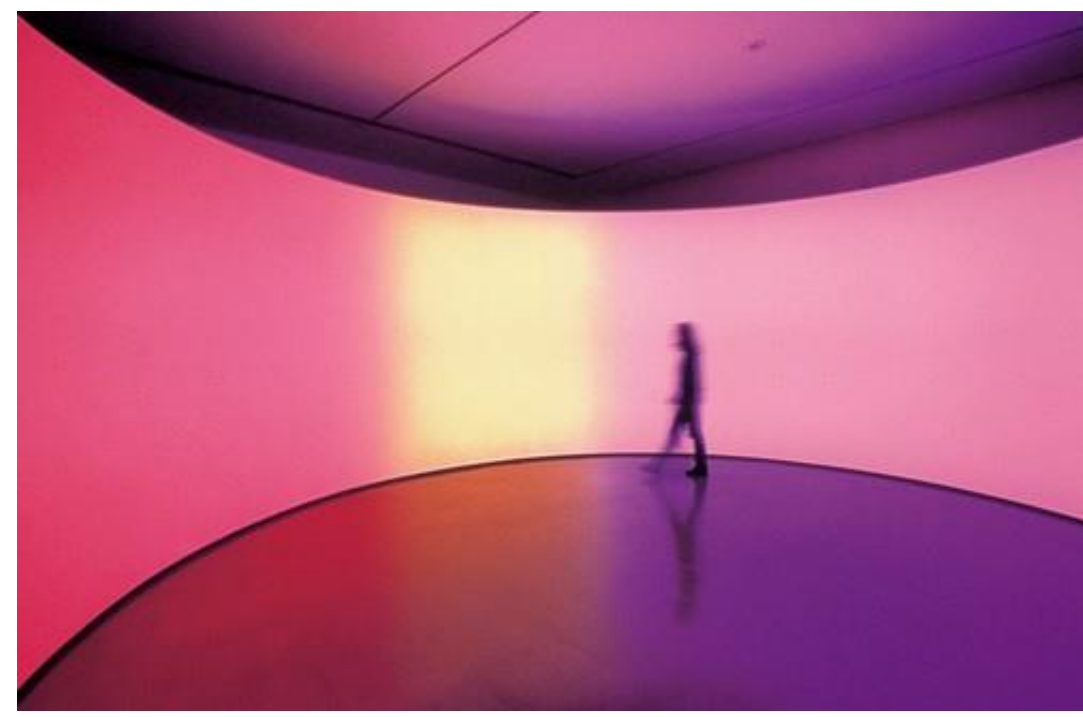

[Fig. 2] Olafur Eliasson $-360^{\circ}$ Room for all Colors (2002). Fuente: Wikiart.org

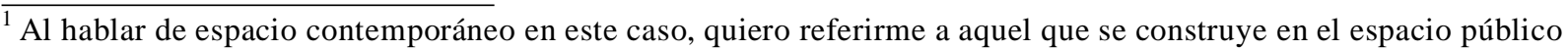
interior, es decir: museos, arquitecturas, galerías u otros que permitan la construcción de una realidad distinta que el espectador pueda transitar y experimentar.
} 
Luz, color y mutabilidad refuerzan la significación de la idea en el espacio, funcionando como metáfora visual inmaterial y afianzando ese carácter poético que activa el proceso de búsqueda de sentido por parte del propio espectador. El espacio se expande planteando incluso una experiencia lingüística a partir de la propia plasticidad del recurso lumínico que, a su vez, requiere de una mayor capacidad cognitiva. Es el movimiento el que permite romper límites en una suerte de hibridación procesual que decodifica un concepto que requiere de una multiplicación de los sentidos implicados en su apreciación estética (Acuna-Zumbado, 2002: 135-153). No se trata de un espacio visual, sino de un espacio de memoria y de sensaciones. Un universo de experiencias en proceso, de sujetos y realidades determinadas por el azar (Úbeda, 2006: 267)

En el espacio contemporáneo la apariencia no se ciñe ya a un objeto sino al lugar entendido como contenedor de experiencias polisensoriales. La percepción del espectador se complica y se amplifica de manera exponencial y se percibe en unas condiciones ambientales determinadas por la luz, el color y la propia construcción del espacio modulado, integrando al espectador y poniendo de manifiesto ese poder significante que afirma su validez como realidad específica dotada de sentido. Se produce una estetización a gran escala de la realidad, apareciendo retóricas de sentido que construyen visiones del mundo con un estatuto ontológico propio que va más allá de lo inmediato (Rodríguez Cunill, 1999: 25-48).

La instalación ocupa un espacio, pero también configura otro virtual, experiencial y artístico con un fuerte carácter referencial que requiere del espectador y su movimiento para totalizar ese entorno proclive a la desmaterialización que, además, se convierte en un sistema abierto en constante conformación. La luz determina los recorridos y configura el espacio, volviéndose soporte modificado metafísicamente. Ese carácter teatral e inmersivo lo complementan los sentidos que consiguen que el acontecimiento ocurra cuando el espectador se incorpora despertando emociones, sentimiento y reflexiones en torno a una idea que se les muestra, revelando unos significados de manera procesual, individual e íntima (Abad, 2011: 8-12). El espacio disuelve todo gesto artístico objetual en una esfera de comunicación que busca producir acciones donde el movimiento se proyecta en el espacio a modo de ritual, donde lo real y lo ideal van de la mano2.

La manipulación y transformación del espacio permite expresar sentimientos e ideas sobre las cuales el público puede transitar. La intención estética y comunicativa del "ente expresivo" impregna la creación, dinamizando y ambientado estos entornos interpretados donde se ofrece la posibilidad de

\footnotetext{
${ }^{2}$ ROCCA VÁSQUEZ, A., Arte conceptual y posconceptual; la idea como arte: Duchamp, Beuys, Cage y Fluxus (1 ${ }^{\mathrm{a}}$ parte) http://www.margencero.com/almiar/arte-conceptual-postconceptual-duchamp-beuys-fluxus-cage/ (consulta: $17 / 08 / 2016)$
} 
transformar al espectador a través del proceso mental que intensifica el sentir y lo conduce hacia el concepto artístico (Gutiérrez, 2009: 132). La escenografía planteada cumple esa función de diálogo que involucra todos los sentidos del participante y que activa estados intelectuales a través de la contemplación al hacerse presente en la propia experiencia corporal (Abad, 2011: 12-23).

La instalación de luz contemporánea como expresión artística busca, no solo espacios físicos, sino también psicológicos, donde el público experimente y comprenda el aspecto temporal que la interacción proporciona3. A través de luces, colores y sombras se revela el propio espacio que actúa sobre la percepción del espectador en términos físicos estimulando las retinas y decodificando la información que se siente como una realidad subjetiva La luz es un vehículo transformador del espacio que crea atmósferas etéreas que se diluyen (Martínez Sellés, 2010: 71). Los artistas de luz tuvieron muy en cuenta todos estos fenómenos. James Turrell o Dan Flavin [3] transforman y descontextualizan el espacio por medio del recurso lumínico estableciendo conexiones entre sus propuestas y el espectador (Paulo Roselló, 2012: 196-197).

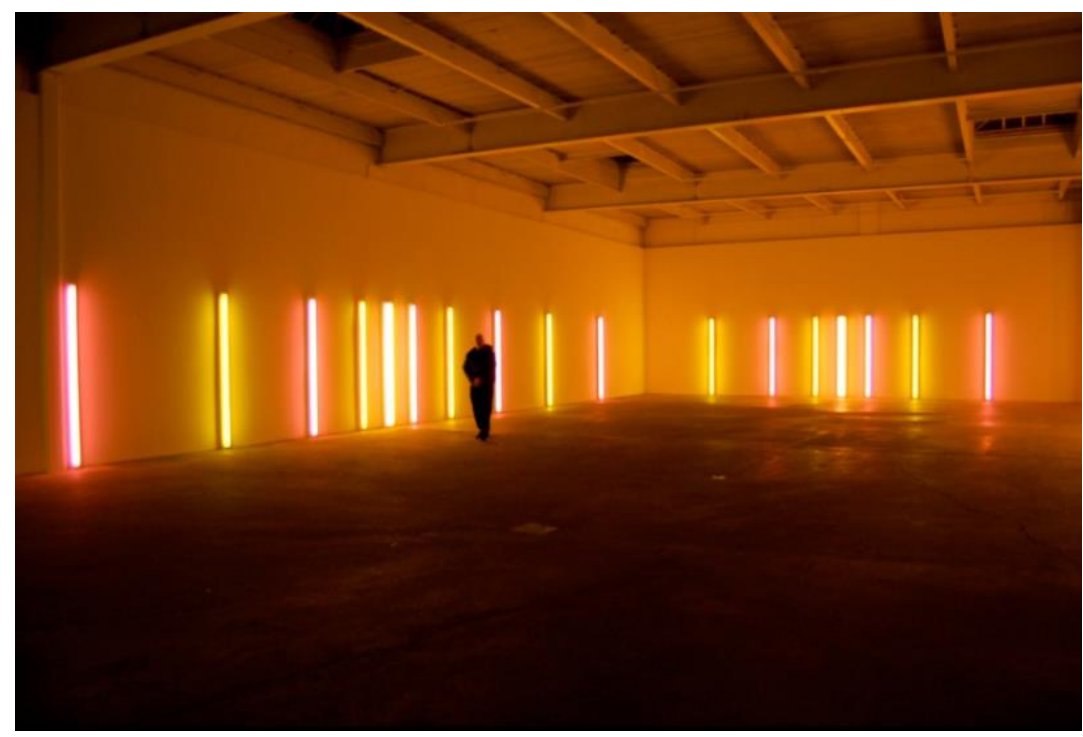

[Fig. 3] Dan Flavin - Alternating Pink and "Gold" (1967). Fuente: Wikiart.org

Dan Flavin presenta un arte de pensar mediante luz y colores de fabricación industrial. Corporiza la luz apelando a los sentidos y a la subjetividad de los ambientes requiriendo de la acción activa (Maderuelo, 2008: 213-314). Es la luz coloreada la que define la estructura espacial, potenciando ese carácter mental que hace visible lo invisible y dialoga con una eternidad de índole metafísica. La luz se materializa y se proyecta en el espacio (Larrañaga 2001: 30-74). Ejemplo de ello es la instalación Alternating Pink and "Gold" (1967) que se referencia en la anterior imagen.

\footnotetext{
${ }^{3}$ Anónimo, Arte contemporáneo. La instalación artística, emblema del arte conceptual. Febrero 2013, https://blogdeartecontemporaneo.wordpress.com/2013/02/26/la-instalacion-artistica-emblema-del-arte-conceptual/ (Consulta: 01/09/2016)
} 
James Turrell plantea propuestas simples y a la vez complejas, donde el público habita un espacio creado a través de su mente y cognición. Sus obras se desmaterializan mediante la luz como lenguaje, pero rompiendo las convenciones tradicionales y la estructura que esta genera. Es un espacio ligado a un sentido simbólico de la luz, pero le interesa más cómo ocupa el espacio, como se modula y las estructuras que genera a modo de metáfora. Son espacios mentales para el espectador que abren “espacios de conciencia” (Paulo Roselló, 2012: 196-205). Como ejemplo se puede ver la instalación realizada en el Museo Guggenheim de Nueva York, Aten Reign (20134) o Rodeen Crater(2012)5.

\subsection{El espacio de luz gótico}

En el espacio gótico, todo el sistema de iluminación y los elementos que forman parte del mismo juegan un papel importante en su configuración, transmitiendo un concepto del que hay que participar para conocer. La luz crea relaciones de significado que permiten al fiel acceder a la metáfora visual que ha de comprender y experimentar. Luz y color alcanzan gran fuerza expresiva y no solo influyen en la arquitectura como tal, sino que construyen un ambiente plástico que asume un papel trascendente (Jantzen, 1970: 79-79).

Son las propias vidrieras [4] las que crean, a modo de caja de luz, una atmósfera coloreada y cambiante donde todos los elementos modifican al son de las horas el espacio, generando vibraciones que transforman la materia en algo ingrávido que baña el interior, arrancando al fiel del mundo y elevándolo a un plano superior, accediendo a su espíritu a través de la cognición y los sentimientos (Dios, 2011: 75-78). Esta atmósfera simbólica se relaciona con lo divino, transformando la luz natural externa en un tipo no-natural que converge con la idea inmaterial que transciende más allá del mundo sensible, transportando al fiel a la idea o concepto que esconde la construcción (Nieto Alcaide, 2006:14-15). Todos los elementos se subordinan al sistema de iluminación que juega un papel de lenguaje plástico creador de un ambiente que el fiel debe recorrer y del que debe participar para alcanzar la idea de Dios.

\footnotetext{
${ }^{4}$ James Turrell - Aten Reign. Museo Guggenheim (Nueva York), http://web.guggenheim.org/exhibitions/turrell/ (Consulta: 09/10/2016).

${ }^{5}$ James Turrell - Roden Crater, http://rodencrater.com/about/ (Consulta: 07/10/2016).
} 


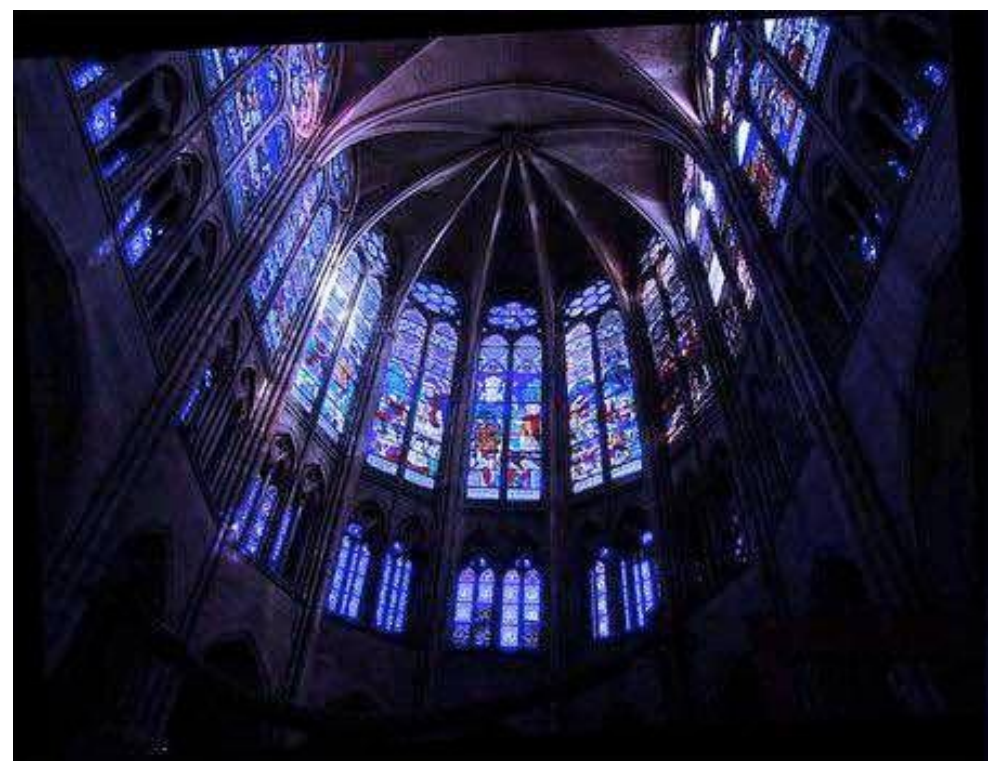

[Fig. 4] Cabecera de la Catedral de Saint Denís. Vitrales azul cobalto. Fuente: Google images

Esta desmaterialización visual se asocia al ámbito espiritual que transciende y que tiene que ver con un componente óptico a partir de lo inmaterial, es decir, la corporeidad de la luz como agente no-visible que se hace carne por medio de la plasticidad artística, donde todo converge y ayuda a revelar la idea abstracta mediante ese efecto de elevación (Sebastián, 2012: 342). La edificación se concibe como un todo a través de este recurso, pero también del muro, que se vuelve translúcido convirtiéndose en vidrio coloreado y que se extiende a toda la estructura (Camille, 2005: 40). La dimensión irreal que se genera debe ser habitada y completada por el fiel, al cual se le revela algo que anteriormente estaba oculto. Esa disolución entre el fiel y la obra muestra ese profundo surgir de la "vivencia" en la que los sentidos se unen, en este caso, desde la perspectiva de lo sagrado y cuyo concepto o idea se relaciona con esa dimensión cósmica o psíquica a la que solo se accede mediante la capacidad reflexiva de lo que allí acontece y el intelecto (Blanco, 2014: 48-51). La obra exhibe su tiempo propio, lejos de ser determinada por una duración infinita: en ella se permanece y se aprehende lo que es la eternidad (Castro, 2003: 595-596).

El espacio gótico, como espacio estético y conceptual, rompe con el régimen visual tradicional. Con los procesos de transformación religiosa y el nuevo planteamiento estético-ideal relacionado con la luz que se da en torno al siglo I a. C, surgen espacios que deben experimentarse, sentirse y recorrerse. La luz y el color necesitaban de la participación del sujeto y su mundo interior para sentir la belleza a través de los sentidos. El concepto teológico del arte y la idea de belleza del cosmos como obra de dios suscitó dudas sobre su posibilidad de representación, así, los presupuestos metafísicos, permitieron traspasar los límites materiales en la búsqueda de un plano sensorial y sublime, así como la configuración de un espacio simbólico y espiritual: la catedral (Tatarkiewicz, 
2007: 5-24). Este espacio provoca al fiel un cortocircuito espiritual metafísico que lo eleva al firmamento sin dejar de ser una simbiosis entre experiencia religiosa y especulación (Tatarkiewicz, 2007: 149-150).

La obra plantea una red de relaciones en torno al propio concepto, donde el espectador representa la progresiva degradación del objeto (Quijano, 2014: 44-46). El edificio pasa a un segundo plano frente a las transformaciones inmateriales que se producen en su interior, las cuales debe experimentar el fiel recorriendo y participando de un espacio donde los límites desaparecen y donde, solo a través de la reflexión, es posible acceder a ese concepto divino superior que completa la metáfora mediante la lectura y la interpretación del signo.

El espacio se convierte en un proceso continuo de recepción, donde la actividad reflexiva trasciende a las escrituras y se percibe como un hecho que se expande apelando a lo trascendente. El espacio se desmaterializa en pos de la idea, convirtiéndose en un sistema abierto donde el comportamiento mental es imprescindible y donde el azar estructura una especie de aura que invita a la exploración estética de aquellos que entran a contemplarla (De Blas Ortega, 2011: 1-20). La catedral encierra un contenido fenomenológico e imaginario donde el fiel capta y siente lo divino en su interior (Ruiz Domènech, 1980: 49-69). La actitud cognoscitiva aquí busca aprehender y percibir esa realidad sobrenatural construida a través del elemento lumínico que a su vez construye escenografías que hablan y dialogan con el fiel, haciendo visible la metáfora ideal que encierra la catedral gótica (Medina del Río et al, 2013: 97). Hay muchísimos ejemplos de catedrales donde se generan ambientes de luz coloreados a través de sus vidrieras como son las catedrales de Notre- Dame, Chartres, Saint-Denis, Mallorca o León [5].

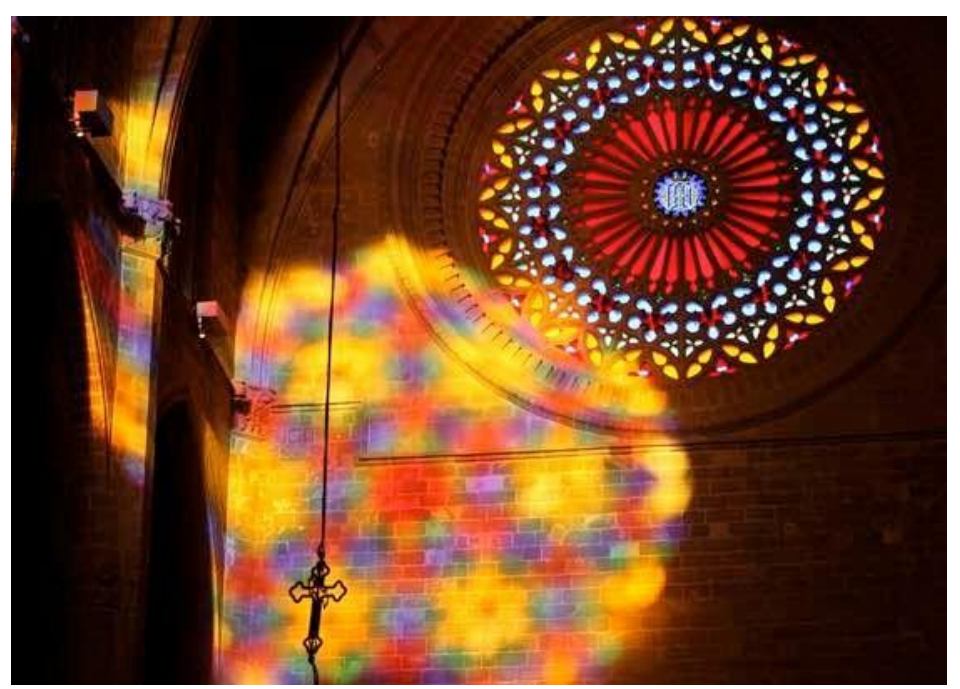

[Fig. 5] Detalle de luz coloreada por el rosetón de la Catedral de Palma de Mallorca. Fuente: Google Images 


\section{Conclusiones}

Tras la realización de este estudio, queda patente que el recurso estético de la luz es capaz de construir espacios polisensoriales a partir de ambientes abstractos que representan metáforas lumínicas, valiéndose de las diferentes teorías y estrategias propias de la luz como lenguaje. Tanto en el espacio lumínico contemporáneo de la instalación artística como en el de la catedral gótica, los escenarios evocan una dimensión poética que posibilita una experiencia estética espacio-temporal con carácter metafísico y, en algunas ocasiones, hasta sagrado.

La luz utilizada como recurso estético implica conceptos simbólicos y filosóficos por su carácter inmaterial, por lo que la experiencia sensorial que se extrae de estas configuraciones espaciales, a través de la misma, contiene diversas referencias que activan la participación del espectador, siendo necesaria esta, junto al conocimiento para permitir, no solo la autocomprensión dentro del propio fenómeno estético sino, también, la autocomprensión del ser dentro de él. Hablamos de espacios híbridos que generan una dialéctica entre lo visible y lo no-visible, pero que se experimentan a través del habitar espacial y que despiertan emociones en el espectador. A través de la luz, como construcción significante, se accederá a ese concepto o idea que nunca se agota, sino que se conjuga entre la teoría y la praxis existencial.

La obra comunica algo que debe comprenderse y que se experimenta a través de la vivencia corporal, la cual atrae los sentidos del participante y lo involucra. Éste establece un diálogo en todo su recorrido, encontrando unidades de sentido que le permiten entender las ideas y planteamientos del reto espacial, alcanzando la comprensión ontológica total del hecho en sí a través de la virtualidad que se expande y resignifica constantemente, transformando la realidad. La configuración de un mundo propio se da en ambas manifestaciones a modo de ritual entre el espectador y el propio ambiente lumínico construido donde se reafirma el espacio y se vive como acontecimiento diferente de lo cotidiano y natural.

La arquitectura, en ambos casos, se desmaterializa y transciende a través de la iluminación, reforzando los volúmenes y los valores cromáticos que juegan también un papel importante en la construcción de estas atmósferas. Si bien en el caso contemporáneo se usan luces artificiales coloreadas de corte tecnológico, en el caso de la catedral, son las propias vidrieras las que generan ese efecto cromático, bañando el interior espacial y definiendo recorridos, dramatizando o fabricando texturas y relieves, amplificando, de esta forma, el acto de comunicación y percepción espiritual. 
Estos espacios utópicos conducen y subliman la realidad, desarrollando un tiempo propio ideal, pero en consonancia con el espacio vivido y experimentado. El espectador se des-ubica y destemporaliza a través de la respuesta fenomenológica del propio cuerpo que desoculta lo oculto de una manera activa frente al concepto. Hablamos en ambos casos de un espacio procesual mediado por la cognición y movimiento del cuerpo. Se produce una actitud estética frente al hecho que es registrada por la propia acción del caminante que se mueve hacia la evocación de la idea, aprehendiendo el concepto a través del intelecto y fabricando una imagen mental como traducción de ese espacio donde la idea, la teoría y el pensamiento se subraya en detrimento de la obra objeto y la materialidad.

Tanto en el espacio contemporáneo de la instalación como en el gótico de la catedral, la operación mental, actuación e intervención del espectador es necesaria para establecer diálogo entre el espacio dado y el propio participante. El público influye en la obra y la experimenta física y mentalmente como proceso, generando una relación no sólo temporal, sino también dinámica y cambiante, en la cual el objeto material desaparece primando la idea de espacio abierto donde el propio espectador, por medio de un proceso receptivo y perceptivo, construye su conocimiento sobre la obra y accede al significado que la envuelve.

Se puede decir por tanto que, en ambos casos, lo material e inmaterial, la ausencia o la presencia del espíritu, lo real o lo imaginario etc., despiertan emociones en el espectador en relación a su cuerpo en movimiento que completa el espacio, convirtiéndolo en una extensión del concepto mental que la luz se encarga de fabricar a través de modulaciones y manipulaciones.

Es un espacio vivido, experimentado, practicado y configurado por la experiencia y la imaginación donde los conceptos de dualidad, simultaneidad e interactividad se vuelven importantes para la comprensión de la metáfora espacial. Esa dimensión espacial y temporal de la que gozan ambas manifestaciones conduce a la afirmación de la experiencia artística y estética del espectador frente a una obra desmaterializada y procesual que construye una estructura ideal como «espacio vivencial» materializado a través del arte. 


\section{Referencias bibliográficas}

ABAD MOLINA, Javier., Experiencia estética y arte de la participación: Juego, símbolo y celebración. Universidad Autónoma de Madrid. Recuperado de: http://www.oei.es/historico/artistica/experiencia_estetica_artistica.pdf(Consulta: 01/09/2016)

ACUNA-ZUMBADO, Eduardo., (2002). Hacia la construcción del sujeto y sus procesos de lectura en la hipertextualidad latinoamericana. University of Kansas: Kansas, MA.

BLANCO, Sara., (2014). El arte y lo sagrado. Su encarnación en la Capilla Rothko en Ilu. Revista de Ciencias de las Religiones, $\mathrm{n}^{\circ}$ 14, Universidad de Granada, pp. 47-64

CAMILLE, Michelle., (2005). Arte Gótico. Visiones Gloriosas. Editorial AKAL S.A: Madrid CASTRO, Sixto. J., (2003). La temporalidad de la obra de arte en Anuario filosófico, XXXVI/3, pp. 587-599.

DE BLAS ORTEGA, Mariano., (2011). Nulla Dies sine linea. El proceso como obra y la obra como proceso continuo. Trabajo final de máster. Universidad complutense de Madrid: Madrid JANTZEN, Hans., (1970). La arquitectura gótica. Editorial Nueva Visión: Buenos Aires MADERUELO, Javier., (2008). La idea de espacio en la arquitectura y el arte contemporáneos 1960- 1989. Editorial AKAL S.A: Madrid

MEDINA DEL RÍO, Juan Manuel y CASINELLO PLAZA, M Josefa., (2013). “La luz gótica. Paisaje religioso y arquitectónico de la época de las catedrales”. Hispania Sacra, LXV, Extra I, enerojunio, pp. 95-98

MENDEZ LLOPIS, Carles., (2013). "El espacio de la inmaterialidad. Del ocultamiento a plena vista de Olafur Eliasson" en Revista el artista, n 10, noviembre, pp. 131-139.

NIETO ALCAIDE, Victor., (2006). La luz, símbolo y sistema visual. Editorial Cátedra: Madrid ROCCA VÁSQUEZ, Adolfo., Arte conceptual y posconceptual; la idea como arte: Duchamp, Beuys, Cage y Fluxus ( $1^{\text {a }}$ parte) Recuperado de: http://www.margencero.com/almiar/arte- conceptualpostconceptual- duchamp-beuys-fluxus-cage/ (consulta: 17/08/2016)

RODRÍGUEZ CUNILL, Inmaculada., (1999). Multiplicidad y fragmentariedad en el arte contemporáneo a través de un análisis de instalaciones y videoinstalaciones. Tesis Doctoral. Universidad de Sevilla: Sevilla.

RUÍZ DOMÈNECH, José Enrique., (1980). El origen de la catedral: estética escolástica e ideología eclesiástica en Revista Medievalia, Vol. 1. Barcelona, pp. 49-82

SEBASTIÁN, Santiago., (2012). Mensaje simbólico del Arte Medieval. Editorial Encuentro: Madrid TATARKIEWICZ, Wladyslaw., (2007). La estética medieval. Historia de la estética. Vol. II.

Editorial AKAL S. A: Madrid 
ÚBEDA FERNÁNDEZ, María Elena., (2006., La Mirada desbordada: el espesor de la experiencia del sujeto estético en el marco de la crisis del régimen escópico. Tesis Doctoral. Editorial de la Universidad de Granada: Granada 